\title{
Systematic Heuristic Evaluation of Computerized Consultation Order Templates: Clinicians' and Human Factors Engineers' Perspectives
}

\author{
April Savoy, $\mathrm{PhD}^{1,2,3}$, Himalaya Patel, $\mathrm{PhD}^{1}$, Mindy E. Flanagan, $\mathrm{PhD}^{4}$, Michael Weiner, $\mathrm{MD}, \mathrm{MPH}^{1,2,4}$, \\ Alissa L. Russ, $\mathrm{PhD}^{1,2,5}$ \\ ${ }^{1}$ Center for Health Information and Communication, US Department of Veterans Affairs, Veterans Health \\ Administration, Health Services Research and Development Service CIN 13-416, Richard L. Roudebush \\ VA Medical Center, Indianapolis, IN, USA; ${ }^{2}$ Regenstrief Institute, Inc., Indianapolis, IN, USA; ${ }^{3}$ Indiana \\ University School of Informatics and Computing, Richmond, IN, USA; ${ }^{4}$ Indiana University School of \\ Medicine, Indianapolis, IN, USA; ${ }^{5}$ Department of Pharmacy Practice, College of Pharmacy, Purdue \\ University, West Lafayette, IN, USA
}

\section{Corresponding author}

April Savoy

+1 7659738333

april.savoy@va.gov

\section{Keywords (Suggested MeSH Terms)}

Referral and Consultation; Heuristics; Electronic Health Records; Human Engineering; Medical Order Entry Systems

\section{Acknowledgments}

We are thankful to the clinicians, research assistants, and clinical application coordinators at the three VA sites that assisted us in data collection. In addition, we are grateful to Jason J. Saleem, PhD for writing the grant proposal and generating study ideas and Laura G. Militello for piloting the methods.

This is the author's manuscript of the article published in final edited form as:

Savoy, A., Patel, H., Flanagan, M. E., Weiner, M., \& Russ, A. L. (2017). Systematic Heuristic Evaluation of Computerized Consultation Order Templates: Clinicians' and Human Factors Engineers' Perspectives. Journal of Medical Systems, 41(8), 129. https://doi.org/10.1007/s10916-017-0775-7 


\section{Abstract}

\section{Objective}

To assess the usability of consultation order templates and identify problems to prioritize in design efforts for improving referral communication.

\section{Methods}

With a sample of 26 consultation order templates, three evaluators performed a usability heuristic evaluation. The evaluation used 14 domain-independent heuristics and the following three supplemental references: 1 new domainspecific heuristic, 6 usability goals, and coded clinicians' statements regarding ease of use for 10 sampled templates.

\section{Results}

Evaluators found 201 violations, a mean of 7.7 violations per template. Minor violations outnumbered major violations almost twofold, 115 (57\%) to 62 (31\%). Approximately 68\% of violations were linked to 5 heuristics: aesthetic and minimalist design (17\%), error prevention (16\%), consistency and standards (14\%), recognition rather than recall (11\%), and meet referrers' information needs (10\%). Severe violations were attributed mostly to meet referrers' information needs and recognition rather than recall. Recorded violations yielded potential negative consequences for efficiency, effectiveness, safety, learnability, and utility. Evaluators and clinicians demonstrated $80 \%$ agreement in usability assessment.

\section{Discussion}

Based on frequency and severity of usability heuristic violations, the consultation order templates reviewed may impede clinical efficiency and risk patient safety. Results support the following design considerations: communicate consultants’ requirements, facilitate information seeking, and support communication.

\section{Conclusion}

While the most frequent heuristic violations involved interaction design and presentation, the most severe violations lacked information desired by referring clinicians. Violations related to templates' inability to support referring clinicians' information needs had the greatest potential negative impact on efficiency and safety usability goals. Heuristics should be prioritized in future design efforts. 


\section{Systematic Heuristic Evaluation of Computerized Consultation Order Templates: Clinicians' and Human Factors Engineers' Perspectives}

\section{Introduction}

As primary care providers' (PCPs) referral of patients to specialists increases in the United States, so does the need for effective communication about referrals.[e.g., 1,2] Particularly difficult is communication about the referred patient, including the patient's clinical history and the referral's purpose, importance, and urgency.[3-8] If information is not conveyed efficiently and effectively, patients' appointments and treatments may be delayed.[4]

A common approach to improving referral communication is to create consultation order (i.e., referral) templates within the electronic health record (EHR). Though intended to prompt necessary diagnostic testing and other clinic-specific prerequisites, these templates lack standardized structure and content across subspecialty units both within and across health care facilities. Consequently, templates may vary in efficiency and effectiveness.[9] For example, although consultation order templates are used in the health care system of the United States Department of Veterans Affairs (VA), about one in three consultation orders is returned to the referring practitioner for further information or new, required diagnostic testing.[10] Ordering consultation is often a first step in referrals. If orders are obstructed by templates with inadequate usability, then addressing these usability problems is likely to improve consultation efficiency and success. One method for measuring usability is a heuristic evaluation, through which experts identify potential usability problems by comparing designs against established principles (i.e., heuristics).[11] Although heuristic evaluation has benefitted other health information technologies [12-20], to our knowledge, consultation order templates have not undergone systematic assessment. As part of a larger study of referrals, our objective was to identify the prevalence, variety, and potential severity of usability problems in consultation order templates.

\section{Methods}

\section{Selection of Consultation Order Templates}

We collected a purposive sample of outpatient templates from three geographically dispersed VA facilities (Appendix, Fig. 1). First, we gathered templates identified by PCPs and interviewed PCPs from 
two VA sites between December 2014 and February 2016.[21] From the interviews, we identified ten templates that PCPs described as either easy or difficult to use (Appendix, Fig. 1). Second, we used consultation completion rates, computed as the number of consultations with a clinician signature divided by the number of consultations ordered during a 12-month period (July 2014-June 2015). Because the template often constitutes a first step in the consultation process, lower completion rates can indicate problematic template design. Completion rates were collected from six specialty clinics (cardiology, mental health, oncology, ophthalmology, orthopedics, and rheumatology) at three VA facilities. We excluded routine procedures (e.g., stress echocardiogram), inter-facility orders, out-of-network (non-VA) consultations, and templates used fewer than 300 times over the 12 months. From the remaining templates, we selected those associated with the lowest completion rates (Appendix, Table 1). This yielded 18 templates with completion rates between 39\% and 84\%. Together, interviews and completion rates yielded a sample of 26 templates.

\section{Usability Heuristics and Goals}

We used 15 usability heuristics. Fourteen were collected from Nielsen (1994) and Shneiderman (2010) (Appendix, Table 2).[11,22] The fifteenth heuristic was created to address clinical decision making; it was given the name meet referrers' information needs.[21] Evaluators also assessed heuristic violations with respect to six usability goals: effectiveness, efficiency, safety, utility, learnability, and memorability (Appendix, Table 3).[23] For each violation, evaluators noted which usability goal was most likely to be impeded.

\section{Evaluation of Template Screenshots}

Following published recommendations to identify the majority of usability violations, we used three evaluators.[11,24] The evaluators were researchers in social psychology, human factors engineering, and informatics. Before the formal evaluation, evaluators underwent 3 hours of practice with five templates and a draft of the evaluation form. Evaluators then met to agree on modifications to the form, interpretations of violations, and documentation terminology.

To counter potential fatigue or carryover effects, each evaluator was assigned templates in a computerrandomized order. Between November 2015 and February 2016, evaluators reviewed blocks of 6 to 10 templates at a time, with 1 to 2 weeks between blocks, until all templates were assessed. Working

independently, evaluators examined screenshots of each template and recorded each violation's most applicable usability heuristic, its most applicable usability goal, its description, and its severity (Appendix, Table 4). Severity ratings were obtained from Nielsen (1994): 1 = cosmetic, 2 = minor, 3 = major, 4 = catastrophic.[11,25] Afterwards, evaluators' results were aggregated; the assigned 
heuristic, severity rating, and associated usability goal were determined by the statistical mode (if available) or by consensus.[26]

\section{Comparison with Primary Care Practitioners' Perceptions}

We qualitatively compared results of the heuristic evaluation to transcripts of PCP interviews. [21] Based on PCPs' descriptions, a qualitative analyst classified templates as either easy or difficult to use. To assess alignment between the heuristic evaluation and interview classifications, we summed minor and major heuristic violations and computed the mean per template. Using this mean, each template was classified as either below average or above average for number of violations. Heuristic evaluation findings and interview data were considered aligned if a template was coded as both easy and below average, or if the template was coded as both difficult and above average by the PCPs and evaluators, respectively.

\section{Results}

Across 26 templates, evaluators identified 201 heuristic violations. Every template violated at least one heuristic. Templates had a mean of 7.7 violations $(S D=3.4)$. The median severity was 2 , range from 1 to 3 (cosmetic, minor, and major). Twenty-four violations (12\%) were cosmetic, and minor violations outnumbered major violations almost twofold, 115 (57\%) to 62 (31\%).

\section{Heuristic Violations}

About 68\% of violations were linked to 5 heuristics: aesthetic and minimalist design (17\%), error prevention (16\%), consistency and standards (14\%), recognition rather than recall (11\%), and meet referrers' information needs (10\%). Fourteen heuristics were violated at least once (Table 1). 
Table 1 Frequency of heuristic violations, by heuristic and severity of violation

\begin{tabular}{lllll}
\hline & \multicolumn{5}{c}{ Severity of violation } \\
Usability heuristic & Cosmetic & Minor & Major & Total \\
\hline Aesthetic and minimalist design & 13 & 21 & 1 & 35 \\
Error prevention & 2 & 22 & 9 & 33 \\
Consistency and standards & 3 & 22 & 3 & 28 \\
Recognition rather than recall & 0 & 6 & 16 & 22 \\
Meet referrers' information needs & 1 & 7 & 11 & 19 \\
Design dialogs to yield closure & 0 & 6 & 5 & 11 \\
Flexibility and efficiency of use & 0 & 11 & 0 & 11 \\
Reduce short-term memory load & 0 & 2 & 7 & 9 \\
Help and documentation & 0 & 5 & 2 & 7 \\
User control and freedom & 0 & 5 & 2 & 7 \\
Cater to universal usability & 4 & 2 & 0 & 6 \\
Visibility of system status & 1 & 5 & 0 & 6 \\
Match between system and the real world & 0 & 0 & 4 & 4 \\
Support internal locus of control & 0 & 1 & 2 & 3 \\
\hline Total & 24 & 115 & 62 & 201 \\
\hline
\end{tabular}

$N=26$ consultation order templates. The only heuristic for which no violations were found was help users recognize, diagnose, and recover from errors.

\section{Most Frequent Violations}

Nearly $85 \%$ of templates $(n=22)$ violated aesthetic and minimalist design. Examples include lengthy instructions and nested lists requiring excessive scrolling (Fig. 1). Additionally, many templates contained a checkbox to "activate" the template. This checkbox was unnecessary because the template had already been selected from a list. Moreover, $69 \%$ of the templates $(n=18)$ violated the heuristic error prevention, most often by allowing conflicting information to be submitted. For example, some questions enabled multiple selection allowing responses to be both "Yes” and “No" (Fig. 2).

\section{[Insert Fig. 1]}

Fig. 1 A violation of the heuristic aesthetic and minimalist design

This template, used for ordering consultations with the cardiology service, violated the heuristic aesthetic and minimalist design because of its lengthy preliminary instructions and nested checkboxes. 


\section{[Insert Fig. 2]}

Fig. 2 A violation of the heuristic error prevention

This template, used for ordering consultations with the oncology service, violated the heuristic error prevention because the checkboxes allowed both "Yes" and "No" responses to the same question.

\section{Most Frequent Major Violations}

Every template had a major violation. Major violations were attributed most frequently to recognition rather than recall (50\% of templates) and meet referrers' information needs (35\% of templates). Violations to recognition rather than recall typically involved entering information into the template either from memory or from other parts of the EHR (Fig. 3). Additionally, these violations were identified when the template did not provide the scope of practice or a list of diagnostic tests preceding the order. Meet referrers' information needs was violated when a template omitted essential information such as pre-requisites or contact information. Urgent requests require referrers to contact the consultant, but some templates omitted consultants’ or clinics' contact information (Fig. 4). A major violation of consistency and standards was noted when the template did not directly prompt for a reason for consultation and/or did not use consistent prompts and response formats (Fig. 5).

\section{[Insert Fig. 3]}

Fig. 3 A violation of the heuristic recognition rather than recall

This template, used for ordering consultations with the ophthalmology service, violated the heuristic recognition rather than recall, because it requested reentry of data from other parts of the EHR (e.g., procedures, diagnoses, and previous clinical encounters).

\section{[Insert Fig. 4]}

Fig. 4 A violation of the heuristic meet referrers' information needs This template, used for ordering consultations with the orthopedics service, violated the heuristic meet referrers' information needs, because its instructions for paging the on-call specialist omitted the pager number or a link to that information.

\section{[Insert Fig. 5]}

Fig. 5 A violation of the heuristic consistency and standards 
These two templates, used for ordering consultations with (a) the rheumatology service and (b) the mental health service, violated the heuristic consistency and standards, because they requested similar information using different prompts and response formats.

\section{Implicated Usability Goals}

Of the six usability goals, efficiency was most often the concern (32\%), followed by safety (24\%), learnability (22\%), effectiveness (11\%), and utility (10\%) (Table 2).

Table 2 Frequency of heuristic violations, by usability goals and type of violation

\begin{tabular}{lllll}
\hline \multirow{2}{*}{ Usability goal } & \multirow{4}{c}{ Cosmetic } & Minor & Major & Total \\
\hline Efficiency & 9 & 34 & 22 & 65 \\
Safety & 2 & 28 & 19 & 49 \\
Learnability & 9 & 32 & 3 & 44 \\
Effectiveness & 0 & 12 & 11 & 23 \\
Utility & 4 & 9 & 7 & 20 \\
\hline Total & 24 & 115 & 62 & 201 \\
\hline
\end{tabular}

$N=26$ order templates. No violations of the memorability goal were identified.

Efficiency violations involved seemingly unnecessary complexity. This included instructions spanning the screen's height and nested checklists requiring iterative review and decision making. Additionally, complex templates required clinicians to copy and paste information from the EHR, such as treatment history and lab results.

Safety violations included incomplete or inaccurate copying and pasting of information from elsewhere in the EHR. Additionally, templates had no standardized nomenclature, which could cause errors in template selection and rejection of the order. Moreover, important exclusion criteria for appropriateness of consults were listed at the end of templates, rather than at the top. Thus, a referrer may finish the template before realizing the patient needs a different consulting service.

Whereas the learnability goal comprised mostly minor violations, both the effectiveness goal and the utility goal had comparable numbers of minor and major violations. Learnability violations involved the software's limited guidance in task performance (e.g., hyperlinks appearing identical to ordinary text; missing instructions). Effectiveness violations involved poor support for task-related actions (e.g., ordering necessary lab tests). Utility violations involved inflexibility in task completion (e.g., not presenting alternative methods to contact the consulting service). 


\section{Comparison of the Heuristic Evaluation with Primary Care Practitioners' Perceptions}

Interviews with PCPs uncovered six templates perceived as difficult and four perceived as easy. Difficult templates required a large amount of scrolling, displayed multiple paragraphs of instructions, or required many tests. In contrast, easy templates were described generally by PCPs as having a shorter length, clear guidelines, or linked order sets.

For the 10 templates identified from PCP interviews, there was $80 \%$ agreement among PCPs and heuristic evaluators (Table 3).

Table 3 Comparison of dichotomized perceptions from PCPs and mean split of violations from heuristic evaluation

\begin{tabular}{lll}
\hline Specialty of template (Site) & PCP perceptions & Rate of heuristic violations \\
\hline Podiatry (B) & Difficult & Above average \\
Dermatology (B) & Difficult & Above average \\
Mental Health (C) & Difficult & Above average \\
Neurosurgery (B) & Difficult & Above average \\
Gastroenterology (C) & Difficult & Above average \\
Nephrology (C) & Easy & Below average \\
Orthopedics (C) & Easy & Below average \\
Cardiology (C) & Easy & Below average \\
Neurology (C)* & Difficult & Below average \\
Endocrine (C)* & Easy & Above average \\
\hline
\end{tabular}

* Indicates disagreement between PCP interviews and the heuristic evaluation.

Agreement was demonstrated across the classifications based on the three prominent aspects discussed (length, guidelines, and orders). For example, both evaluators and PCPs identified long templates as inefficient: Evaluators asserted violations of the aesthetic and minimalist design heuristic, and PCPs consistently mentioned that long templates required more time to complete. One PCP stated the following.

Dermatology here has five separate fields for the history, and all of them are mandatory fields.

So instead of being able to write a little paragraph summarizing what the skin condition is, I have to enter five separate mandatory fields and type it in.

Another example was the neurosurgery template, where a PCP said, “Their template asks a bazillion questions. It doesn't always import the data, so you have to back out into the chart and start over....” 
One source of disagreement among the evaluators and PCPs related to guidelines for referring. Evaluators associated clear, concise guidelines with meet referrers' information needs heuristic, but they were unable to detect what types of medical terms may be too specialized for PCPs. As a result, few violations were noted for the neurology template, whereas a PCP remarked, "You don’t need all the 18-syllable words... just tell me what you want in plain English.”

\section{Discussion}

To our knowledge, this is the first study to evaluate consultation order templates systematically against design heuristics and provide evidence to inform design. Overall, heuristics captured important usability issues in the templates. Moreover, the meet referrers' information needs heuristic and PCP interviews provided insights about cognitive needs and contextual use. In total, 201 violations were identified by heuristic evaluators and supported by clinicians’ accounts. To address efficiency and safety usability goals, the five heuristics—aesthetic and minimalist design, error prevention, consistency and standards, recognition rather than recall, and meet referrers' information needs - that were linked to most frequent and severe violations should be the focus of redesign efforts. The $80 \%$ agreement among evaluators and clinicians is a strong indicator that our results capture both perspectives.

The most frequent causes of heuristic violations in this study were driven by design flaws in interaction and presentation, which are often highlighted with heuristic evaluations.[27] Repetitive violations included inconsistent organization of form elements (e.g. buttons, directions, text boxes). These types of violations could be linked to the specific software used to implement the examined EHR and usability guidelines during the development process. Although templates are reviewed based on clinical needs, VA's health care system has no apparent enterprise-wide design standards addressing template usability. In addition, the implemented EHR software restricts consultation order templates to unformatted text (e.g., no tables, font emphasis, or graphics) and basic form input elements (color and icons are not supported). Auto-population is used sometimes but seldom for clinically relevant information.

Both VA and non-VA studies have noted information-transfer challenges that fragmented care and delayed access to specialty care.[4,5,8,9,28] The most severe heuristic violations in this study were attributed to inaccessible or omitted information, and there is evidence that this occurs across multiple healthcare systems. Compared with design- and interaction-related heuristic violations, informationrelated heuristic violations are more broadly applicable across templated order forms. Although VA and non-VA templates have different development processes and software capabilities, their referral requests are similar in that templates are typically used for consultation orders or referral letters, and the templates require various specific types of information (e.g., relevant patient history and diagnostic test results).[29- 
32] Supporting examples identified in this study include omission of consultants' contact information, missing or hidden prerequisites, clinical information requirements causing navigation outside the template, and rigid, structured data entry that limited referrers' explanation of the reason for the consultation. Specific challenges with consultation orders described across VA and non-VA studies relate to unclear reasons for consultation request,[5-7] incomplete prerequisites,[4,10] requests sent to the wrong clinics,[10] and missing information.[3,4,9,5,8,28,33]

This study extends our knowledge about how EHR interface issues contribute to challenges in the consultation process, a core component of patient care.[8,34] We identified prioritized heuristics and key considerations for new or redesigned templates. We expect these results to inform efforts to improve consultation orders. First, specialty clinics' requirements for consultation appropriateness and specific diagnostic testing should be concisely communicated to PCPs, either in the template or via a link to reference information. Second, to limit excessive, redundant navigation to other parts of the EHR for relevant clinical information already contained in the medical record, the relevant clinical details could be automatically displayed in, or adjacent to, the template's text. As previously recommended, such autopopulated fields reduce the data-entry burden for PCPs and may decrease the possibility of errors.[28] Third, templates should minimize the amount of other information that referrers are required to enter manually. Templates should, however, support referrers' need to communicate information that is not included in the template requirements, perhaps through free-text fields or mechanisms to communicate directly with consultants. Similarly, Esquivel et al. (2012) recommended a combination of structured and free-text fields to capture necessary information and provide flexibility for additional clinical details, respectively.[28] Although heuristic evaluations do not generate immediate solutions to usability problems, our evaluation identified and described violations to guide design changes that may improve consultation order usability and related care processes.

Limitations of our study should be considered. Our study focused on consultation templates from the largest integrated health care system in the United States. Although we did not formally review templates from other health care systems' EHRs, we found no evidence of inapplicability. Our sampling methods were intended to include templates at both extremes of usability, not a cross-section representing all templates. None of the heuristic evaluators had formal clinical training, although evaluators gained insight on the cognitive requirements of referrers through field observations in the larger study. Due to security policies, evaluators examined templates via screenshots, with minimum functionality demonstrated.[35] This may explain the lack of observed violations for the heuristic help users recognize, diagnose, and recover from errors. Finally, this study focused on only one step of the consultation process: templates associated with consultation orders. Solving the larger problem of referral management will require 
further research that examines other steps in the consultation process, including triage, scheduling, and follow-up.[8]

\section{Conclusion}

With a heuristic evaluation, we identified, quantified, and prioritized templates' usability problems. The majority of the recorded heuristic violations (57\%) were attributed to cosmetic (12\%) and minor design flaws (57\%). Furthermore, at least one major violation was recorded for each template, which accounted for the remaining $31 \%$ of the total violations. These high severity violations related to templates' inability to support referring clinicians' information needs and had the greatest potential negative impact on efficiency and safety usability goals. We recommend heuristic evaluation as a low-cost method of inspecting usability in the early stages of design. We suggest two enhancements from the literature: merging sets of domain-independent heuristics and adding domain-specific heuristics.[11,25] Based on our results, template design efforts should prioritize the five heuristics discussed with emphasis on the following: concisely communicate consulting clinics' requirements, limit unnecessary navigation to other parts of the EHR, and support referrers' need to communicate information outside the provided structured input fields. Results can inform a user-centered design process to improve consultations and create more innovative approaches that mediate effective communication among clinicians who are coordinating a patient's care. Future research should include formal usability testing with clinicians. If usability issues for initiating consultations are addressed, we expect a decrease in the delays in accessing specialty care.

\section{Compliance With Ethical Standards}

\section{Funding}

This study was supported by VA HSR\&D Grant IIR 12-102 (Principal Investigator: Dr. M. Weiner) and the Center for Health Information and Communication, US Department of Veterans Affairs, Veterans Health Administration, Health Services Research and Development Service, CIN 13-416, Richard L. Roudebush VA Medical Center, Indianapolis, Indiana. Dr. Russ was supported by a VA HSR\&D Career Development Award, \#11-214. M. Weiner is Chief of Health Services Research and Development at the Richard L. Roudebush VA Medical Center in Indianapolis, Indiana. The views expressed in this article are those of the authors and do not necessarily represent the views of the Department of Veterans Affairs.

\section{Conflict of Interest}

The authors have no competing interests to declare. 


\title{
Ethical Approval
}

This article contains no studies with human participants or animals.

\section{Contributorship}

\begin{abstract}
A.W. Savoy and H. Patel designed the heuristic evaluation, collected and analyzed data, and drafted the manuscript. M.E. Flanagan coauthored the funded grant proposal and collected and analyzed data. M. Weiner and A.L. Russ coauthored the funded grant proposal, and A.L. Russ proposed the manuscript on heuristic evaluation. All authors interpreted the findings, made critical revisions, and approved the published manuscript; all authors agree to be accountable for all aspects of the work in ensuring that questions related to the accuracy or integrity of any part of the work are appropriately investigated and resolved.
\end{abstract}




\section{References}

1. Kirsh SR, Ho PM, Aron DC (2014) Providing specialty consultant expertise to primary care: An expanding spectrum of modalities. Mayo Clin Proc 89 (10):1416(1411).

doi:10.1016/j.mayocp.2014.04.016

2. Barnett ML, Song Z, Landon BE (2012) Trends in physician referrals in the United States, 1999-2009.

Arch Intern Med 172 (2):163-170. doi:10.1001/archinternmed.2011.722

3. Mehrotra A, Forrest CB, Lin CY (2011) Dropping the baton: Specialty referrals in the United States.

Milbank Q 89 (1):39-68. doi:10.1111/j.1468-0009.2011.00619.x

4. Zuchowski JL, Rose DE, Hamilton AB, Stockdale SE, Meredith LS, Yano EM, Rubenstein LV, Cordasco KM (2015) Challenges in referral communication between VHA primary care and specialty care. J Gen Intern Med 30 (3):305-311. doi:10.1007/s11606-014-3100-x

5. O'Malley AS, Reschovsky JD (2011) Referral and consultation communication between primary care and specialist physicians: Finding common ground. Arch Intern Med 171 (1):56-65.

doi:10.1001/archinternmed.2010.480

6. Conley J, Jordan M, Ghali WA (2009) Audit of the consultation process on general internal medicine services. Quality \& Safety in Health Care 18 (1):59-62. doi:10.1136/qshc.2007.025486

7. Salerno SM, Hurst FP, Halvorson S, Mercado DL (2007) Principles of effective consultation: An update for the 21st-century consultant. Arch Intern Med 167 (3):271-275. doi:10.1001/archinte.167.3.271

8. Saleem JJ, Russ AL, Neddo A, Blades PT, Doebbeling BN, Foresman BH (2011) Paper persistence, workarounds, and communication breakdowns in computerized consultation management. Int J Med Inform 80 (7):466-479. doi:10.1016/j.ijmedinf.2011.03.016

9. Hysong SJ, Esquivel A, Sittig DF, Paul LA, Espadas D, Singh S, Singh H (2011) Towards successful coordination of electronic health record based-referrals: A qualitative analysis. Implement Sci 6 (1):84. doi:10.1186/1748-5908-6-84

10. Singh H, Esquivel A, Sittig DF, Murphy D, Kadiyala H, Schiesser R, Espadas D, Petersen LA (2011)

Follow-up actions on electronic referral communication in a multispecialty outpatient setting. J Gen Intern Med 26 (1):64-69. doi:10.1007/s11606-010-1501-z

11. Nielsen J (1994) Heuristic evaluation. In: Nielsen J, Mack RL (eds) Usability Inspection Methods. vol 1. Wiley, New York, NY, pp 25-62

12. Thyvalikakath TP, Schleyer TKL, Monaco V (2007) Heuristic evaluation of clinical functions in four practice management systems: A pilot study. J Am Dent Assoc 138 (2):209-218.

doi:10.14219/jada.archive.2007.0138

13. Edwards PJ, Moloney KP, Jacko JA, Sainfort F (2008) Evaluating usability of a commercial electronic health record: A case study. Int J Hum Comput Stud 66 (10):718-728. doi:10.1016/j.ijhcs.2008.06.002 14. Chan J, Shojania KG, Easty AC, Etchells EE (2011) Usability evaluation of order sets in a computerised provider order entry system. BMJ Qual Saf 20 (11):932-940. doi:10.1136/bmjqs.2010.050021

15. Nabovati E, Vakili-Arki H, Eslami S, Khajouei R (2014) Usability evaluation of laboratory and radiology information systems integrated into a hospital information system. J Med Syst 38 (4):35.

doi:10.1007/s10916-014-0035-z

16. Phansalkar S, Zachariah M, Seidling HM, Mendes C, Volk L, Bates DW (2014) Evaluation of medication alerts in electronic health records for compliance with human factors principles. J Am Med Inform Assoc 21 (e2):e332-e340. doi:10.1136/amiajnl-2013-002279

17. Balatsoukas P, Williams R, Davies C, Ainsworth J, Buchan I (2015) User Interface Requirements for Web-Based Integrated Care Pathways: Evidence from the Evaluation of an Online Care Pathway Investigation Tool. J Med Syst 39 (11):183. doi:10.1007/s10916-015-0357-5 
18. Lilholt PH, Jensen MH, Hejlesen OK (2015) Heuristic evaluation of a telehealth system from the Danish TeleCare North Trial. Int J Med Inform 84 (5):319-326. doi:10.1016/j.ijmedinf.2015.01.012 19. van Engen-Verheul MM, Peute LWP, de Keizer NF, Peek N, Jaspers MWM (2016) Optimizing the user interface of a data entry module for an electronic patient record for cardiac rehabilitation: A mixed method usability approach. Int J Med Inform 87:15-26. doi:10.1016/j.ijmedinf.2015.12.007

20. Xu L, Wen D, Zhang X, Lei J (2016) Assessing and comparing the usability of Chinese EHRs used in two Peking University hospitals to EHRs used in the US: A method of RUA. Int J Med Inform 89:32-42. doi:10.1016/j.ijmedinf.2016.02.008

21. Wu J, Militello LG, Flanagan ME, Barker BC, Rehman S, Porter BW, Adams JM, Savoy AW, Russ AL, Weiner M (2016) Barriers and facilitators to using electronic health records for referrals between primary and specialty care clinics. Paper presented at the AMIA 2016 Annual Symposium, Chicago, IL, 2016

22. Shneiderman B (2010) Guidelines, principles, and theories In: Shneiderman B, Plaisant C, Cohen M, Jacobs $S$ (eds) Designing the User Interface: Strategies for Effective Human-Computer Interaction. 5 edn. Addison-Wesley, Boston, MA, pp 73-112

23. Preece J, Sharp H, Rogers Y (2015) What is interaction design? In: Interaction Design: Beyond Human-Computer Interaction. 4th ed. edn. Wiley, Chichester, United Kingdom, pp 19-22

24. Savoy A, Yinni G, Salvendy G (2009) Effects of Importance and Detectability of Usability Problems on Sample Size Requirements. Int J Hum Comput Interact 25 (5):430-440. doi:10.1080/10447310902865024 25. Zhang J, Johnson TR, Patel VL, Paige DL, Kubose T (2003) Using usability heuristics to evaluate patient safety of medical devices. J Biomed Inform 36 (1-2):23-30. doi:10.1016/S1532-0464(03)00060-1 26. Saldaña J (2015) An introduction to codes and coding. In: The Coding Manual for Qualitative Researchers. 3 edn. Sage, Los Angeles, CA,

27. Tarrell A, Grabenbauer L, McClay J, Windle J, Fruhling AL (2015) Toward improved heuristic evaluation of EHRs. Health Systems 4 (2):138-150. doi:10.1057/hs.2014.19

28. Esquivel A, Sittig DF, Murphy DR, Singh H (2012) Improving the effectiveness of electronic health record-based referral processes. BMC Med Inform Decis Mak 12 (1):107. doi:10.1186/1472-6947-12-107 29. Chen AH, Murphy EJ, Yee HF, Jr. (2013) eReferral - A new model for integrated care. N Engl J Med 368 (26):2450-2453. doi:10.1056/NEJMp1215594

30. Tuot DS, Leeds K, Murphy EJ, Sarkar U, Lyles CR, Mekonnen T, Chen AH (2015) Facilitators and barriers to implementing electronic referral and/or consultation systems: a qualitative study of 16 health organizations. BMC Health Serv Res 15:568. doi:10.1186/s12913-015-1233-1

31. Straus SG, Chen AH, Yee HF, Jr., Kushel MB, Bell DS (2011) Implementation of an electronic referral system for outpatient specialty care. Paper presented at the AMIA Annual Symposium, 32. Warren J, White S, Day KJ, Gu Y, Pollock M (2011) Introduction of electronic referral from community associated with more timely review by secondary services. Appl Clin Inform 2 (4):546-564. doi:10.4338/ACl-2011-06-RA-0039

33. Chen AH, Yee HF, Jr. (2009) Improving the primary care-specialty care interface: Getting from here to there. Arch Intern Med 169 (11):1024-1026. doi:10.1001/archinternmed.2009.140

34. Flanagan ME, Saleem JJ, Millitello LG, Russ AL, Doebbeling BN (2013) Paper- and computer-based workarounds to electronic health record use at three benchmark institutions. J Am Med Inform Assoc 20 (e1):e59-e66. doi:10.1136/amiajnl-2012-000982

35. Allen M, Currie LM, Bakken S, Patel VL, Cimino JJ (2006) Heuristic evaluation of paper-based Web pages: A simplified inspection usability methodology. J Biomed Inform 39 (4):412-423.

doi:10.1016/j.jbi.2005.10.004 


\section{Appendix}

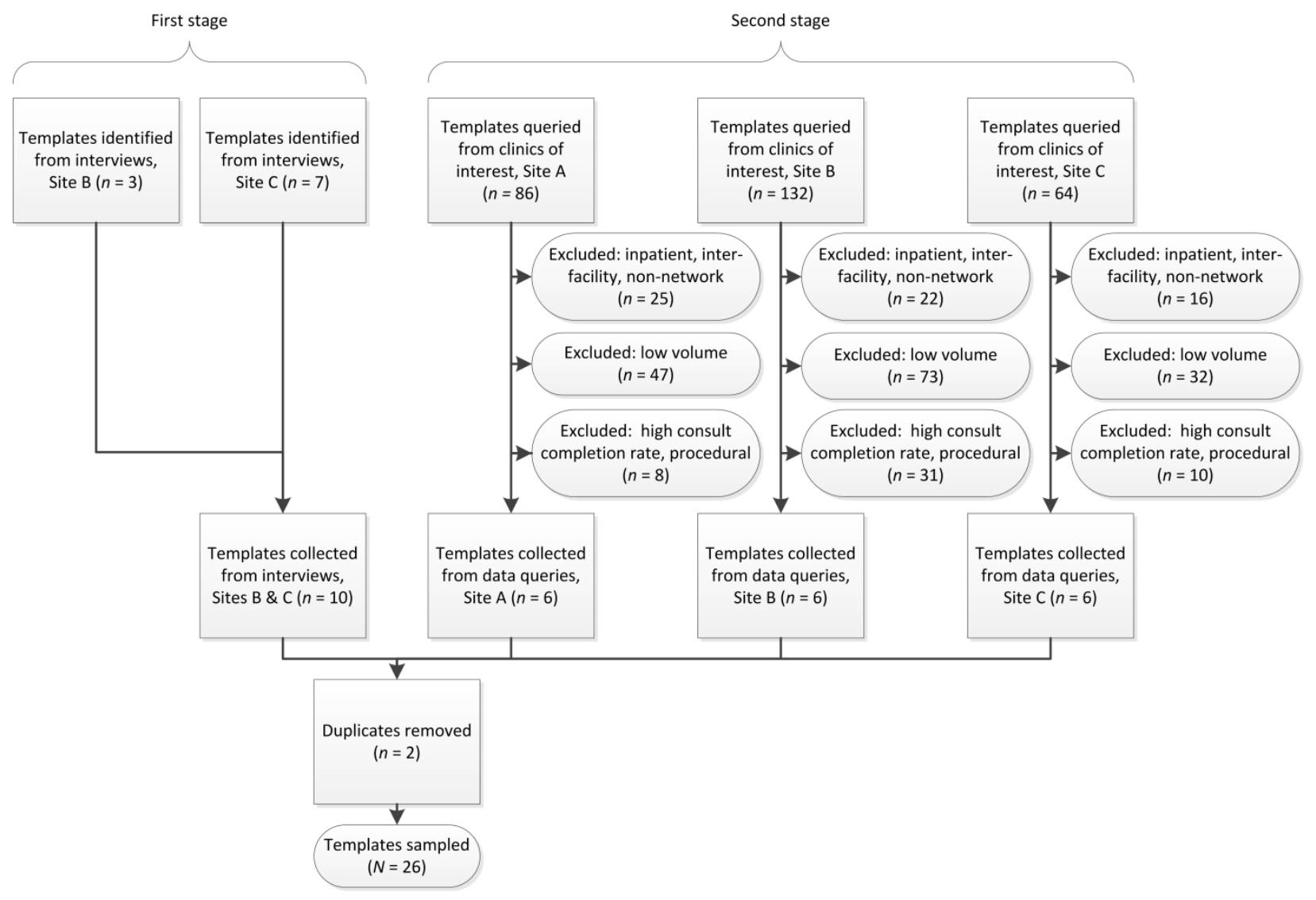

Fig. 6 Sampling of templates for heuristic evaluation

Templates were identified through interviews with primary care practitioners at Sites B and C and through database queries at Sites A, B, and C. 
Table 4 Consultation completion rates

\begin{tabular}{lrr}
\hline Template name (Site) & Consultation orders submitted & Consultations completed (\%) \\
\hline Psychiatry (A) & 522 & 204 (39) \\
Mental Health (B) & 1125 & 496 (44) \\
Rheumatology (B) & 318 & $172(54)$ \\
Mental Health (C) & 1003 & $594(59)$ \\
Hematology/Oncology (C) & 1070 & $661(62)$ \\
Rheumatology (A) & 691 & $430(62)$ \\
Cardiology (B) & 1020 & $649(64)$ \\
Orthopedics Shoulder (C) & 1500 & $964(64)$ \\
Ophthalmology (A) & 4842 & $3254(67)$ \\
Cardiology (C) & 1436 & $995(69)$ \\
Ophthalmology (C) & 715 & $523(73)$ \\
Oncology (A) & 464 & $345(74)$ \\
Orthopedics (B) & 814 & $615(76)$ \\
Ophthalmology (B) & 1474 & $1137(77)$ \\
Oncology (B) & 398 & $308(77)$ \\
Cardiology (A) & 1667 & $1331(80)$ \\
Rheumatology (C) & 517 & $418(81)$ \\
Orthopedics (A) & 2373 & $2000(84)$ \\
\hline
\end{tabular}

Completion rates for 18 of the consultation order templates included in the heuristic evaluation.

Table 5 Usability heuristics[11,22]

\begin{tabular}{|c|c|}
\hline Usability heuristic & Definition \\
\hline Recognition rather than recall & $\begin{array}{l}\text { Minimize the user's memory load by making objects, actions, and options visible. } \\
\text { The user should not have to remember information from one part of the dialogue to } \\
\text { another. Instructions for use of the system should be visible or easily retrievable } \\
\text { whenever appropriate. }\end{array}$ \\
\hline $\begin{array}{l}\text { Meet referrers' information } \\
\text { needs }\end{array}$ & $\begin{array}{l}\text { Referrers' cognitive requirements to complete the consultation order template } \\
\text { effectively, and to communicate information necessary for the consultation. Users } \\
\text { should be able to send and receive information with high fidelity, rather than having } \\
\text { to find or create workarounds. }\end{array}$ \\
\hline Error prevention & $\begin{array}{l}\text { Even better than helpful error messages is a careful design that prevents errors and } \\
\text { other problems. Either eliminate error-prone conditions or check for them, and } \\
\text { present users with a confirmation option before they commit to the action. }\end{array}$ \\
\hline $\begin{array}{l}\text { Reduce short-term memory } \\
\text { load }\end{array}$ & $\begin{array}{l}\text { Humans' limited capacity for information processing in short-term memory requires } \\
\text { that designers avoid interfaces in which users must remember information from one } \\
\text { screen and then use that information on another screen. }\end{array}$ \\
\hline Design dialogs to yield closure & $\begin{array}{l}\text { Sequences of actions should be organized into groups with a beginning, middle, and } \\
\text { end. Informative feedback at the completion of a group of actions gives operators } \\
\text { the satisfaction of accomplishment, a sense of relief, a signal to drop contingency } \\
\text { plans from their minds, and an indicator to prepare for the next group of actions. For } \\
\text { example, e-commerce web sites move users from selecting products to the checkout, }\end{array}$ \\
\hline
\end{tabular}


Match between system and the real world

Consistency and standards

Help and documentation

User control and freedom

Support internal locus of control

Aesthetic and minimalist design

Flexibility and efficiency of use

Cater to universal usability

Visibility of system status

Prevent errors*

Help users recognize, diagnose, and recover from errors

Offer informative feedback* ending with a clear confirmation page that completes the transaction. Although referrers can see their consultation orders on demand, the confirmation is not clearly provided to them automatically.

The system should speak the users' language, with words, phrases and concepts familiar to the user, rather than system-oriented terms. Follow real-world conventions, making information appear in a natural and logical order.

Users should not have to wonder whether different words, situations, or actions have the same meaning. Follow platform conventions.

Although lack of need for documentation is ideal, providing help and documentation may be needed. Any such information should be easy to locate and search, be focused on the user's task, list concrete steps to be carried out, and not be too large.

Users often choose system functions erroneously, and will need a clearly marked "emergency exit" to leave the unwanted state without having to go through an extended series of steps and computerized dialogue. Support undo and redo.

Experienced users strongly desire the sense that they are in charge of the interface and that the interface responds to their actions. They don't want surprises or changes in familiar behavior, and they are annoyed by tedious data-entry sequences, difficulty in obtaining necessary information, and inability to produce their desired result.

Dialogues should not contain irrelevant or rarely needed information. Every irrelevant unit of information in a dialogue competes with relevant units of information and diminishes their relative visibility.

Accelerators, unseen by the novice user, may speed the process for the expert user, such that the system can cater to both inexperienced and experienced users. Enable users to expedite or tailor frequent actions.

Recognize the needs of diverse users, and design for plasticity, facilitating transformation of content. Novice-to-expert differences, age ranges, disabilities, and technological diversity enrich the spectrum of requirements that guide design.

Adding features for novices, such as explanations, and features for experts, such as shortcuts and faster pacing, can enrich the interface design and improve perceived system quality.

The system should always keep users informed, through appropriate, timely feedback.

As much as possible, design the system such that users cannot make serious errors. If a user makes an error, the interface should detect the error and offer simple, constructive, and specific instructions for recovery. Erroneous actions should leave the system state unchanged, or the interface should give instructions about restoring the state.

Error messages should be expressed in plain language, precisely indicate the problem, and constructively suggest a solution.

For every user action, system feedback should occur. For frequent and minor actions, the response can be modest, whereas for infrequent and major actions, the response should be more substantial. Visual presentation of the objects of interest provides a convenient environment for showing changes explicitly. 
Permit easy reversal of actions* As much as possible, actions should be reversible. This feature relieves anxiety, since the user knows that errors can be undone, and exploration of unfamiliar options is encouraged. The units of reversibility may be a single action, a data-entry task, or a group of actions.

Strive for consistency*

Consistent sequences of actions should be required in similar situations; identical terminology should be used in prompts, menus, and help screens; and consistent color, layout, capitalization, and fonts should be used throughout. Exceptions should be comprehensible and limited in number.

*Violations were recoded for four pairs of heuristics with similar definitions. Strive for consistency was recoded as consistency and standards; offer informative feedback was recoded as visibility of system status; prevent errors was recoded as error prevention; and permit easy reversal of actions was recoded as user control and freedom.

Table 6 Usability goals[23]

\begin{tabular}{ll}
\hline Usability goal & Description \\
\hline Effectiveness & Does the interface do what it is designed to do? \\
Efficiency & Once learned, does the interface prevent unnecessary work? Are users productive? \\
Safety & Does the interface minimize errors, especially severe ones, and facilitate recovery from \\
& errors? \\
Utility & Does the interface help users to complete tasks using their preferred approach? \\
Learnability & Does the interface promote discovery of functions, both basic and advanced? \\
Memorability & Do users recall how to complete their tasks, especially infrequent ones? \\
\hline
\end{tabular}

Table 7 Heuristic evaluation form with sample observation

\begin{tabular}{lllll}
\hline Template & Usability dimension & Most applicable heuristic & Problem description & Severity \\
\hline S1 & Utility (helpful?) & Meet referrers' information & $\begin{array}{l}\text { Users are instructed } \\
\text { to telephone specific usability }\end{array}$ \\
& needs & $\begin{array}{l}\text { staff, but telephone } \\
\text { problem: fixing }\end{array}$ \\
& & $\begin{array}{l}\text { numbers are not } \\
\text { provided should be } \\
\end{array}$ & & given low \\
& & priority \\
\hline
\end{tabular}

